\section{MS5-P41 Structure and function of microcystin and CP12-CBS in toxic cyanobacteria}

Claudia Hackenberg ${ }^{1}$, Elke Dittmann ${ }^{2}$, Victor Lamzin ${ }^{1}$

1. European Molecular Biology Laboratory, c/o Desy, Hamburg, Germany

2. Institute for Biochemistry and Biology, University of Potsdam, Germany

email: hackenberg@embl-hamburg.de

Microcystins (MC) are the most common and notorious toxins produced by harmful cyanobacterial blooms in marine, brackish and fresh water habitats. They strongly inhibit protein phosphatases type 1 and $2 \mathrm{~A}$ in the liver by covalently binding to cysteines in the active side $[1,2]$, causing severe and often lethal hepatic necrosis. It is assumed that $\mathrm{MC}$ increase the resistance of toxic cyanobacteria towards various environmental stresses $[3,4]$, enabling the toxic species to become dominant. Detailed knowledge about the actual molecular function of MC is, however, still fairly limited.

We aim to follow up on the hypothesis that MC binds to the cysteine pairs of proteins and acts as protein-modulating metabolite [4] by investigating its effect on two known target proteins: the small regulatory Calvin cycle protein 12 (CP12) and the photosynthetic protein phosphoribulokinase (PRK) of Microcystis. These proteins are of particular interest, because: 1) the activity of PRK is regulated by $\mathrm{CP} 12$ in a redox-dependent manner [5]; 2) cyanobacteria possess a variety of several CP12-variants, a.o. curiously being fused to a CBS domain (Bateman domain) [6]. The CBS domain is found in various organisms, where it is fused to a wide range of proteins regulating their activity. Mutations of the CBS domain are implicated in several hereditary human diseases [7]. Its function in cyanobacteria, particularly as fusion protein with CP12, is so far unknown.

The fusion of CP12 with CBS suggests a functional connection as well as different, multiple roles of CP12 in cyanobacteria. The interaction of CP12 and PRK with microcystin may even add a further layer of complexity in the regulation, stability and activity of these proteins in harmful cyanobacteria. Using interaction studies and macromolecular crystallisation we aim to explore: 1) the role of the CBS domain in CP12;2) whether CP12-CBS is still regulating PRK; 3 ) the binding site of microcystin on PRK and CP12; and 4) if microcystin is affecting the stability, conformation and activity of CP12-CBS and PRK.

[1] Goldberg et al., Nature 376:745-753 (1995)

[2] Xing et al., Cell 127:341-353 (2006)

[3] Dziallas \& Grossart, PLoS ONE 6:e25569 (2011)

[4] Zilliges et al., PLoS ONE 6:e17615 (2011)

[5] Tamoi et al., Proc. Natl. Acad. Sci. USA, 42:504-513 (2005)

[6] Stanley et al., Plant J., 161:824-835 (2013)

[7] Scott et al., J. Clin. Invest. 113:274-284 (2004)
MS5-P42 Biochemical and structural studies of substrate recognition and reaction mechanism of M23 metallopeptidases

Izabela Sabala ${ }^{1}$, Elżbieta Jagielska ${ }^{1}$, Maja Grabowska ${ }^{1}$, Honorata Czapinska $^{1}$, Matthias Bochtler ${ }^{1,2}$

1. International Institute of Molecular and Cell Biology, Warsaw, Poland

2. Institute of Biochemistry and Biophysics, Polish Academy of Sciences, Warsaw, Poland

email: izabela@iimcb.gov.p1

Peptidoglycans build a thick layer around each cell providing a solid envelop around the cell and securing its integrity. They have to be rigid to stand the internal pressure and at the same time flexible to allow cell expansion and division. The rigidity of the cell wall is provided by the thick network of peptidoglycans while its flexibility by orchestrated action of peptidoglycan hydrolases. Each bond in peptidoglycans is believed to be cut by a specific hydrolase. Many of them have been biochemically and structurally characterized but despite enormous efforts a basic questions on their mechanism of action or determination of specificity remains unanswered. Composition and structure of peptidoglycan is species specific and in the case of Staphylococcus is characterized by presence of pentaglycine cross-bridges, which are cleaved by endopeptidases belonging to M23 family of metallopeptidases, like an autolysin LytM and a bacteriocine lysostaphin. These two enzymes have the same specificity and catalytic domains of over $50 \%$ of aa identity but they differ in their role and activity regulation.By combining biochemical and structural methods we have gained new insights into structural bases of peptidoglycan recognition and mechanism of action of M23 family represented by lysostaphin and LytM. We have crystallized and analyzed structures of (1) full length lysostaphin, (3) catalytic domain of lysostaphin and the (3) LytM catalytic domain in complex with transition state analogue. These analysis supported by biochemical tests allow us to propose and discuss the possible mechanism of hydrolysis for M23 peptidases and defined some of the structural features determining substrate specificity.This information is important not only for our deeper understanding of basic biological processes but might also have a great impact on application of theses enzymes in combating staphylococcal infections. Lysostaphin has been successfully tested in various biomedical applications but the main limitation of its use is naturally occurring resistance caused by incorporation of serine into the pentaglycine bridges. The same applies to LytM demonstrating effectiveness as antibacterial agent. Our research might provide information necessary for engineering enzyme specificity overcoming the existing resistance mechanism.

Keywords: M23 peptidases, peptidoglycan hydrolases, lysostaphin, LytM, staphylococcus 